\title{
Topological Invariants and Ground-State Wave functions of Topological Insulators on a Torus
}

\author{
Zhong Wang ${ }^{1,2, *}$ and Shou-Cheng Zhang ${ }^{1,3}$ \\ ${ }^{1}$ Institute for Advanced Study, Tsinghua University, Beijing 100084, China \\ ${ }^{2}$ Collaborative Innovation Center of Quantum Matter, Beijing 100871, China \\ ${ }^{3}$ Department of Physics, Stanford University, Stanford, California 94305, USA
}

(Received 22 August 2013; published 21 January 2014)

\begin{abstract}
We define topological invariants in terms of the ground-state wave functions on a torus. This approach leads to precisely defined formulas for the Hall conductance in four dimensions and the topological magnetoelectric $\theta$ term in three dimensions, and their generalizations in higher dimensions. They are valid in the presence of arbitrary many-body interactions and disorder. These topological invariants systematically generalize the two-dimensional Niu-Thouless-Wu formula and will be useful in numerical calculations of disordered topological insulators and strongly correlated topological insulators, especially fractional topological insulators.
\end{abstract}

DOI: 10.1103/PhysRevX.4.011006

Subject Areas: Condensed Matter Physics, Strongly Correlated Materials,

Topological Insulators

\section{INTRODUCTION}

Topological insulators are among the major recent developments in condensed matter physics [1-3]. The physics of topological insulators started with noninteracting systems [4-15], for which simple and calculable topological invariants have been invaluable tools. More recently, it became clear that the interplay between topology and many-body interactions is a still-richer field [16-59]; therefore, it is highly desirable to develop topological invariants that are valid in the presence of strong interactions.

The root state of three-(spatial)-dimensional (3D) and two-(spatial)-dimensional (2D) topological insulators with time-reversal symmetry is the four-(spatial)-dimensional (4D) quantum Hall $(\mathrm{QH})$ state $[11,60]$ from which the topological field theory of 3D and 2D insulators can be obtained by the procedure of "dimensional reduction" [11]. The electromagnetic effective action of the 4D QH effect reads $[3,61]$

$$
S_{\text {eff }}=\frac{\sigma_{4 D}}{24 \pi^{2}} \int d t d^{4} x \epsilon^{\mu \nu \rho \sigma \tau} A_{\mu} \partial_{\nu} A_{\rho} \partial_{\sigma} A_{\tau},
$$

where we have adopted the units in which the electric charge $e$, the Planck constant $h$, and the light velocity $c$ are all unity. The coefficient $\sigma_{4 \mathrm{D}}$ is referred to as the "4D Hall conductance" (or the 4D Hall coefficient). Physically, the 4D QH effect has the nonlinear topological electromagnetic response [11] $j^{\mu}=\frac{\delta S_{\text {eff }}}{\delta A_{\mu}}=$ $\frac{\sigma_{4 \mathrm{D}}}{8 \pi^{2}} \epsilon^{\mu \nu \rho \sigma \tau} \partial_{\nu} A_{\rho} \partial_{\sigma} A_{\tau}$ in the bulk, which is described naturally

\footnotetext{
*wangzhongemail@gmail.com
}

Published by the American Physical Society under the terms of the Creative Commons Attribution 3.0 License. Further distribution of this work must maintain attribution to the author(s) and the published articles title, journal citation, and DOI. by the Chern-Simons effective action. If a nontrivial 4D QH insulator is cut open in one direction, there are $\left|\sigma_{4 \mathrm{D}}\right|$ copies of 3D chiral-fermion (Weyl-fermion) modes localized at the boundary. These boundary modes are close analogues of the $1 \mathrm{D}$ chiral edge states $[62,63]$ of $2 \mathrm{D} \mathrm{QH}$. In fact, the $\mathrm{QH}$ effect can be generalized to all even spatial dimensions, whose boundary modes are chiral fermions in odd spatial dimensions.

In the noninteracting limit, the explicit formula for $\sigma_{4 \mathrm{D}}$ has been obtained by Qi, Hughes, and Zhang as [11]

$$
\sigma_{4 \mathrm{D}}=c_{2} \equiv \frac{1}{32 \pi^{2}} \int d^{4} k \epsilon^{i j k l} \operatorname{Tr} f_{i j} f_{k l}
$$

where $f_{i j}$ is the non-Abelian Berry curvature defined in terms of the noninteracting Bloch states [64].

Now, a natural question arises: Can we find a formula for $\sigma_{4 \mathrm{D}}$ that is precisely defined in the presence of arbitrary interaction and disorder? Such a formula, if it exists, will be especially desirable for the investigation of fractional quantum Hall states in 4D. More importantly, it may also shed light on strongly interacting topological insulators in lower dimensions.

The same question also arises for the 3D topological insulators, whose effective topological response theory is given by [11]

$$
\begin{aligned}
S_{\text {eff }} & =\frac{1}{8 \pi^{2}} \int d t d^{3} x \epsilon^{i j k l} \theta \partial_{i} A_{j} \partial_{k} A_{l} \\
& =\frac{1}{4 \pi^{2}} \int d t d^{3} x \theta \mathbf{E} \cdot \mathbf{B} .
\end{aligned}
$$

This topological effective action describes the quantized topological magnetoelectric effect, in which an electric field induces a magnetization with a universal constant of proportionality [11]. 
In the noninteracting limit, $\theta$ has a simple expression $[11,65,66]$

$$
\left.\theta=\frac{1}{4 \pi} \int d^{3} k \epsilon^{i j k} \operatorname{Tr}\left\{\left[\partial_{i} a_{j}(k)+\frac{2}{3} i a_{i}(k) a_{j}(k)\right)\right] a_{k}(k)\right\},
$$

which is a 3D Chern-Simons term. In the presence of timereversal symmetry, this Chern-Simons term is quantized and has been shown to be equivalent [66] to the $Z_{2}$ topological invariant [12]. The natural question is as follows: Is there a formula for $\theta$ that is valid in the presence of an arbitrary interaction and disorder? From the experimentalist's perspective, this question is more urgent than the 4D QH case because many 3D topological insulators have been realized in experiments, and the electron-electron interaction has been playing a more important role.

To partially answer these questions, interacting topological invariants expressed in terms of Green's functions at zero frequency (namely, the "topological Hamiltonian" [67]) for interacting insulators have been proposed [68-70], which provide an efficient approach for topological invariants of various topological insulators and superconductors (see, e.g., Refs. [40,50-56,71-75] for applications). However, there are several shortcomings of this approach. First, it cannot be directly applied to disordered systems in which the momentum $\mathbf{k}$ in the singleparticle Green's function is not a good quantum number [76]. Second, it is unclear whether or not that approach will fail for some fractional topological states.

In Ref. [77], Niu, Thouless, and Wu found, for the 2D $\mathrm{QH}$, a topological invariant (the first Chern number) expressed in terms of the ground-state wave function, which is valid in the presence of an arbitrary interaction and disorder. To search for the general formulas for $\sigma_{4 \mathrm{D}}$ in $4 \mathrm{D}$ and $\theta$ in $3 \mathrm{D}$, a hopeful approach is to generalize their formulas to higher dimensions. However, as we will see later, the most straightforward 4D generalization of their formulas, namely, the generalization of the $2 \mathrm{D}$ phase twisting $\left(\theta_{1}, \theta_{2}\right)$ to the $4 \mathrm{D}$ phase twisting $\left(\theta_{1}, \theta_{2}, \theta_{3}, \theta_{4}\right)$ [see Eq. (30)], cannot produce the 4D Hall conductance $\sigma_{4 \mathrm{D}}$. Because of this difficulty, it is unclear how this approach can be generalized to higher-dimensional topological states.

In this paper, we propose general topological invariants for higher-dimensional topological insulators in terms of ground-state wave functions. The boundary conditions adopted here are not the same as the standard one used in Ref. [77], which is a pure gauge with vanishing field strength. Using these new boundary conditions (see Secs. II and VIII, we obtain for $\sigma_{4 \mathrm{D}}$ and $\theta$ simple formulas expressed in terms of the ground-state wave function on a torus [see Eqs. $(12,29,41,44)$, etc]. We also generalize these formulas to higher dimensions [see Eq. (24), etc]. These topological invariants are valid in the presence of an arbitrary interaction and disorder; thus, they can be applied to topological states with strong disorders and strongly correlated topological states including fractionalized states. Unexpectedly, the generalized formula for $\sigma_{4 D}$ appears not as a second Chern number but as the difference between two first Chern numbers [Eqs. (12,29)]. Similarly, the formula for $\theta$ does not appear as a Chern-Simons term but as the difference between two winding numbers [Eqs. (41,44)].

The rest of this paper is organized as follows. In Sec. II, we study the 4D QH and define the topological invariant for integer QH in 4D. In Sec. III, we test this topological invariant in two noninteracting models. We then generalize the 4D topological invariant to higher-dimensional QH effects in Sec. IV. In Sec. V, we present the topological invariants for fractional quantum Hall effects. A different boundary condition is investigated in Sec. VI, which leads to a 4D topological invariant unrelated to the 4D Hall conductance. The next two sections, namely, Secs. VII and VIII, are devoted to $1 \mathrm{D}$ and 3D $\theta$ terms, respectively.

\section{THE 4D HALL COEFFICIENTS $\sigma_{4 \mathrm{D}}$ EXPRESSED IN TERMS OF THE GROUND-STATE WAVE FUNCTION}

In this section, we describe the topological invariant defined in terms of the ground-state wave function of a 4D insulator on a torus with generalized twisted boundary conditions. For simplicity, in this section, we assume that the ground state is unique, while the cases with groundstate degeneracy will be studied in Sec. V. We take the system to be a $4 \mathrm{D}$ torus with circumference $L_{1}, L_{2}, L_{3}, L_{4}$ along the $x_{1}, x_{2}, x_{3}, x_{4}$, directions, respectively. We take the generalized twisted boundary condition parametrized by $\left(\theta_{1}, \theta_{2}, \phi\right)$ as follows [78]. First, for $i=1,2$,

$$
\begin{aligned}
& \Psi\left(\mathbf{r}_{1}, \cdots, \mathbf{r}_{k}+L_{i} \hat{\mathbf{x}}_{i}, \cdots, \mathbf{r}_{N} ; \theta_{1}, \theta_{2}, \phi\right) \\
& \quad=\exp \left(i \theta_{i}\right) \Psi\left(\mathbf{r}_{1}, \cdots, \mathbf{r}_{k}, \cdots, \mathbf{r}_{N} ; \theta_{1}, \theta_{2}, \phi\right),
\end{aligned}
$$

where $\mathbf{r}_{k}$ is the coordinate of the $k$ th particle (other arguments such as spin are not shown here for simplicity of notation), $N$ is the total particle number, and $\hat{\mathbf{x}}_{i}$ is the unit vector along the $x_{i}$ direction. This condition is the same as the one adopted in Ref. [77]. Second,

$$
\begin{aligned}
& \Psi\left(\mathbf{r}_{1}, \cdots, \mathbf{r}_{k}+L_{3} \hat{\mathbf{x}}_{3}, \cdots, \mathbf{r}_{N} ; \theta_{1}, \theta_{2}, \phi\right) \\
& \quad=\exp \left(-i \phi \frac{x_{4}}{L_{4}}\right) \Psi\left(\mathbf{r}_{1}, \cdots, \mathbf{r}_{k}, \cdots, \mathbf{r}_{N} ; \theta_{1}, \theta_{2}, \phi\right) .
\end{aligned}
$$

Since $x_{4} \equiv x_{4}+L_{4}$ on the torus, the flux $\phi$ has to be quantized as $n \phi_{0}$, where the unit flux $\phi_{0} \equiv 2 \pi$, and $n$ is an integer. Lastly,

$$
\begin{gathered}
\Psi\left(\mathbf{r}_{1}, \cdots, \mathbf{r}_{k}+L_{4} \hat{\mathbf{x}}_{4}, \cdots, \mathbf{r}_{N} ; \theta_{1}, \theta_{2}, \phi\right) \\
\quad=\Psi\left(\mathbf{r}_{1}, \cdots, \mathbf{r}_{k}, \cdots, \mathbf{r}_{N} ; \theta_{1}, \theta_{2}, \phi\right) .
\end{gathered}
$$


Physically, these twisted boundary conditions tell us that there is a gauge potential $A_{i}=\theta_{i} / L_{i}$ along the $x_{i}(i=1,2)$ direction and a gauge potential $A_{3}=-\phi \frac{x_{4}}{L_{3} L_{4}}$ along the $x_{3}$ direction; in other words, there is a magnetic flux $\phi$ inside any $2 \mathrm{D}$ torus $T_{34}$ whose coordinates are $\left(X_{1}, X_{2}, x_{3}, x_{4}\right)$ with fixed $\left(X_{1}, X_{2}\right)$.

Before proceeding to our central results, let us briefly outline the motivations of the boundary conditions given in Eqs. (5), (6), and (7). The first motivation is that the $\left(\theta_{1}, \theta_{2}, \theta_{3}, \theta_{4}\right)$ boundary condition [see Sec. VI] does not produce the $4 \mathrm{D}$ Hall conductance. The second motivation is the intuitive relation between the 4D Hall effect and the 2D Hall effect. In Eq. (1), if we take $A_{3}, A_{4}$ to be independent of $x_{0}, x_{1}, x_{2}$, and at the same time take $A_{0}, A_{1}, A_{2}$ to be independent of $x_{3}, x_{4}$, then there is a "dimensional reduction" [79] of the 4D Chern-Simons term to the 2D ChernSimons term: $\sigma_{4 \mathrm{D}} \epsilon^{\mu \nu \rho \sigma \tau} A_{\mu} \partial_{\nu} A_{\rho} \partial_{\sigma} A_{\tau} \rightarrow \sigma_{4 \mathrm{D}} B_{34} \epsilon^{\mu \nu \rho} A_{\mu} \partial_{\nu} A_{\rho}$ (up to a numerical factor), where $B_{34} \equiv \partial_{3} A_{4}-\partial_{4} A_{3}$, and the indices $\mu, \nu, \rho$ in " $\epsilon$ " $\epsilon^{\mu \nu}$ " take the values $0,1,2$. According to this argument, in our boundary conditions given in Eqs. $(5,6)$, and (7), we have taken $\partial_{3} A_{4}-$ $\partial_{4} A_{3}=\phi / L_{3} L_{4}$; thus, we have the dimensional reduction $\sigma_{4 \mathrm{D}} \epsilon^{\mu \nu \rho \sigma \tau} A_{\mu} \partial_{\nu} A_{\rho} \partial_{\sigma} A_{\tau} \rightarrow \sigma_{4 \mathrm{D}} \phi \epsilon^{\mu \nu \rho} A_{\mu} \partial_{\nu} A_{\rho}$. Intuitively, we have the evident identity

$$
\frac{\partial}{\partial \phi}\left(\sigma_{4 \mathrm{D}} \phi \epsilon^{\mu \nu \rho} A_{\mu} \partial_{\nu} A_{\rho}\right)=\sigma_{4 \mathrm{D}} \epsilon^{\mu \nu \rho} A_{\mu} \partial_{\nu} A_{\rho} .
$$

Since the right-hand side of this equation is a 2D ChernSimons term, it seems that we can calculate $\sigma_{4 D}$ using well-known results of 2D quantum Hall effects. In practice, however, it is impossible to take the derivative with respect to $\phi$ because $\phi$ is quantized; i.e., $\phi$ takes only discrete values. To resolve this difficulty, we will take a difference instead of a derivative (see below).

Now our task is to formulate these intuitive arguments as a precise mathematical framework. We can define the Berry connection

$$
a_{i}\left(\theta_{1}, \theta_{2}, \phi\right)=-i\left\langle\Psi\left(\theta_{1}, \theta_{2}, \phi\right)\left|\partial_{\theta_{i}}\right| \Psi\left(\theta_{1}, \theta_{2}, \phi\right)\right\rangle
$$

and the Berry curvature

$$
F_{i j}\left(\theta_{1}, \theta_{2}, \phi\right)=\frac{\partial a_{j}}{\partial \theta_{i}}-\frac{\partial a_{i}}{\partial \theta_{j}},
$$

from which we can define a first Chern number

$$
C(\phi)=\frac{1}{2 \pi} \int_{0}^{2 \pi} d \theta_{1} d \theta_{2} F_{12}\left(\theta_{1}, \theta_{2}, \phi\right),
$$

where we have chosen the notation " $C$ " instead of " $C_{1}$ " to distinguish $C$ from the first Chern number appearing in the 2D quantum Hall effects [77].

With these preparations, the general formula for $\sigma_{4 \mathrm{D}}$ appearing in Eq. (1) is proposed as

$$
\sigma_{4 \mathrm{D}}=C\left(\phi_{0}\right)-C(0) \text {. }
$$

The first term is the Chern number with a unit flux $\phi_{0} \equiv 2 \pi$ in $T_{34}$, and the second term is the Chern number without this flux; in other words, Eq. (12) measures the jump of the first Chern number after inserting a flux $\phi_{0}$ in $T_{34}$. The necessity of the second term $C(0)$ in Eq. (12) can be easily appreciated in a noninteracting model [see Eq. (19)], to be presented in Sec. III. It is also useful to note that $C(0)$ may be zero if the ground state has certain symmetries. For instance, if there is time-reversal symmetry, we have $C(0)=0$ and $\sigma_{4 \mathrm{D}}=C\left(\phi_{0}\right)$.

Equation (12) is expressed in terms of the Berry phase of ground-state wave functions on a torus, which is well defined in the presence of an arbitrary interaction and disorder [80]. Equation (12) can also be written equivalently as

$\sigma_{4 \mathrm{D}}=\frac{1}{2 \pi} \int_{0}^{2 \pi} d \theta_{1} d \theta_{2}\left[F_{12}\left(\theta_{1}, \theta_{2}, \phi_{0}\right)-F_{12}\left(\theta_{1}, \theta_{2}, 0\right)\right]$.

Equations (12) and (13) are among the central equations of the present paper.

Several remarks about Eq. (12) are in order. The noninteracting topological invariant for the 2D quantum Hall effect, namely, the Thouless-Kohmoto-Nightingale-den Nijs (TKNN) invariant [81], is expressed as the first Chern number in the Brillouin zone. The Niu-Thouless$\mathrm{Wu}$ formula [77], as a generalization of the TKNN invariant, is again a first Chern number. Given the second Chern number in Eq. (2) for the 4D noninteracting quantum Hall effect, we may try to express the 4D Hall coefficient $\sigma_{4 \mathrm{D}}$ as a second Chern number on a certain parameter space, for an interacting system. However, this attempt turns out to be unfruitful. Instead, the topological invariant defined in Eq. (12), which gives $\sigma_{4 \mathrm{D}}$, is the difference between two first Chern numbers.

Let us conclude this section with a side remark that the Laughlin's gauge argument [82] can also be generalized to $4 \mathrm{D} \mathrm{QH}$. The boundary conditions in the $x_{3}, x_{4}$, direction are the same as given by Eqs. (6) and (7), but the system is open along the $x_{2}$ direction. When we do the adiabatic evolution $\theta_{1} \rightarrow \theta_{1}+2 \pi$, the charge transferred from the boundary $x_{2}=0$ to $x_{2}=L_{2}$ is denoted as $\Delta Q(\phi)$. The Hall conductance is given as $\sigma_{4 \mathrm{D}}=\Delta Q\left(\phi_{0}\right)-\Delta Q(0)$.

\section{THE NONINTERACTING LIMIT: TWO SIMPLE MODELS}

In this section, we will check in two simple noninteracting models [Eqs. (14) and (19)] that Eq. (12) gives the same result as Eq. (2), as should be the case in the noninteracting limit. Incorporating well-known results of topological classification of noninteracting insulators, we will show that Eq. (12) reduces to Eq. (2) for all noninteracting 4D insulators. 
First, let us consider a noninteracting Hamiltonian for 4D QH [11],

$$
h(\mathbf{k})=v \sum_{i=1}^{4} \sin k_{i} \Gamma^{i}+M(\mathbf{k}) \Gamma^{0},
$$

where $M(\mathbf{k})=m+4-\sum_{i=1}^{4} \cos k_{i}, v$ and $m$ are parameters of the Hamiltonian, and $k_{i} \in[0,2 \pi]$ is the $i$ th momentum of the free Bloch state (the lattice constant has been taken as unity). Here, the Gamma matrices satisfy the identities $\left\{\Gamma^{\mu}, \Gamma^{\nu}\right\}=2 \delta^{\mu \nu}$. For our convenience, we choose the representation $\Gamma^{1}=\tau^{1}, \Gamma^{2}=\tau^{2}, \Gamma^{3}=\tau^{3} \sigma^{1}$, $\Gamma^{4}=\tau^{3} \sigma^{2}, \Gamma^{0}=\tau^{3} \sigma^{3}$.

Instead of solving the model numerically in real space, which is less illuminating for our purpose, let us perform the calculation in the limit where $|m|$ is significantly smaller than unity. In this limit, we can keep only the $\mathbf{k}$-linear terms near $\mathbf{k}=0$, and the Dirac Hamiltonian reads

$h(\mathbf{k}) \approx v\left(k_{1} \tau^{1}+k_{2} \tau^{2}\right)+\tau^{3}\left(v k_{3} \sigma^{1}+v k_{4} \sigma^{2}+m \sigma^{3}\right)$.

In the presence of twisted boundary conditions, the momenta should be replaced by $k_{i} \rightarrow-i D_{i}=$ $-i\left(\partial_{i}-A_{i}\right)$. Let us calculate the first term $C\left(\phi_{0}\right)$ of Eq. (12) for the Dirac Hamiltonian in Eq. (15). In this linear-k limit, we can first solve the Hamiltonian $h^{\prime}\left(k_{3}, k_{4}\right)=v k_{3} \sigma^{1}+v k_{4} \sigma^{2}+m \sigma^{3}$, whose eigenvalues read [83]

$$
E_{0}=m, \quad E_{n \pm}= \pm \sqrt{m^{2}+2 n B v^{2}}(n=1,2, \cdots),
$$

where $B=\phi_{0} / L_{3} L_{4}$. The corresponding eigenwavefunctions are $\left(\psi_{0}, 0\right)^{T}$ and $\left(\psi_{n}, \pm \psi_{n-1}\right)^{T}$, where $\psi_{n}$ is the wave function of the $n$th Landau level of Schrodinger particles [83], whose precise forms do not concern us here. It is useful to note that when $m=0$, the existence of the zero mode $E_{0}$ is guaranteed by the Atiyah-Singer index theorem. Inputting the eigenvalues given in Eq. (16) into the second set of parentheses in Eq. (15), we have the 2D Hamiltonians

$$
\begin{aligned}
h_{0} & =v\left(k_{1} \tau^{1}+k_{2} \tau^{2}\right)+m \tau^{3} ; \\
h_{n \pm} & =v\left(k_{1} \tau^{1}+k_{2} \tau^{2}\right)+E_{n \pm} \tau^{3}(n=1,2, \cdots) .
\end{aligned}
$$

The value of $C\left(\phi_{0}\right)$ can be obtained as the summation of the first Chern number of $h_{0}$ and $h_{n \pm}$, namely, $\frac{1}{2}\left[\operatorname{sgn}\left(E_{0}\right)+\sum_{n} \sum_{\alpha= \pm} \operatorname{sgn}\left(E_{n \alpha}\right)\right]=\frac{1}{2} \operatorname{sgn}(m)$, thanks to the fact that the ground-state wave functions are a Slate determinant of Bloch states in the noninteracting cases. In this calculation, we have not been careful about the high-energy regularization; thus, we can only assert that $C\left(\phi_{0}\right)=\frac{1}{2} \operatorname{sgn}(m)+$ constant. Since we require $C\left(\phi_{0}\right)=0$ as $m \rightarrow+\infty$, we have $C\left(\phi_{0}\right)=\frac{1}{2}[\operatorname{sgn}(m)-1]$. Similarly, we can obtain $C(0)=0$; therefore, we have

$$
\sigma_{4 D}=C\left(\phi_{0}\right)-C(0)=\frac{1}{2}[\operatorname{sgn}(m)-1],
$$

which is the same as $c_{2}$ obtained [11] from Eq. (2) (see also Ref. [84] for calculations for a different model using charge pumping).

Let us move to the second noninteracting model, which will explain the reason why we must include the second term $C(0)$ in Eq. (12). The simple model has the free Hamiltonian

$$
\begin{aligned}
h(\mathbf{k})= & v\left(\sin k_{1} \tau^{1}+\sin k_{2} \tau^{2}\right) \\
& +\left(m+2-\cos k_{1}-\cos k_{2}\right) \tau^{3},
\end{aligned}
$$

which is independent of $k_{3}$ and $k_{4}$. If we take $m=-0.1$, then it is obvious that both $C\left(\phi_{0}\right)$ and $C(0)$ are nonzero; however, they are equal, and therefore $\sigma_{4 D}=C\left(\phi_{0}\right)-$ $C(0)=0$. From Eq. (2), it is obvious that we have $\sigma_{4 D}=c_{2}=0$; therefore, Eqs. (12) and (2) produce the same result in this example.

Although we have only explicitly checked that Eq. (12) reduces to Eq. (2) in Dirac models, it is possible to make a more general statement that Eq. (12) is always equivalent to Eq. (2) in the noninteracting limit. In fact, as has been shown in Refs. $[15,85]$, there is a Dirac-Hamiltonian representative in each class of the 4D QH insulators, which means that any noninteracting Hamiltonian for the 4D insulator can always be smoothly connected to a Dirac Hamiltonian. Therefore, equivalence between Eqs. (12) and (2) in the Dirac model implies their equivalence for all noninteracting Hamiltonians. In the presence of interactions, however, Eq. (2) loses definition, while Eq. (12) remains useful.

\section{QUANTUM HALL EFFECT IN $d=2 l+2$ SPATIAL DIMENSIONS}

Equation (12) can be generalized to $d=2 l+2$ spatial dimensions. The boundary conditions for the $\left(x_{1}, x_{2}\right)$ direction given in Eq. (5) are unchanged, while the boundary conditions for other directions are defined as

$$
\begin{aligned}
& \Psi\left(\mathbf{r}_{1}, \cdots, \mathbf{r}_{k}+L_{2 j+1} \hat{\mathbf{x}}_{2 j+1}, \cdots, \mathbf{r}_{N} ; \theta_{1}, \theta_{2}, \phi_{1}, \cdots, \phi_{l}\right) \\
& \quad=\exp \left(-i \phi_{j} \frac{x_{2 j+2}}{L_{2 j+2}}\right) \Psi\left(\mathbf{r}_{1}, \cdots, \mathbf{r}_{k}, \cdots, \mathbf{r}_{N} ; \theta_{1}, \theta_{2}, \phi_{1}, \cdots, \phi_{l}\right)
\end{aligned}
$$

and

$$
\begin{aligned}
& \Psi\left(\mathbf{r}_{1}, \cdots, \mathbf{r}_{k}+L_{2 j+2} \hat{\mathbf{x}}_{2 j+2}, \cdots, \mathbf{r}_{N} ; \theta_{1}, \theta_{2}, \phi_{1}, \cdots, \phi_{l}\right) \\
& \quad=\Psi\left(\mathbf{r}_{1}, \cdots, \mathbf{r}_{k}, \cdots, \mathbf{r}_{N} ; \theta_{1}, \theta_{2}, \phi_{1}, \cdots, \phi_{l}\right)
\end{aligned}
$$

for $j=1,2, \cdots l$. Physically, these conditions means that there is a flux $\phi_{j}$ in the 2D torus $T_{2 j+1,2 j+2}$. We can define the Berry connection 


$$
\begin{aligned}
& a_{i}\left(\theta_{1}, \theta_{2}, \phi_{1}, \cdots, \phi_{l}\right) \\
& \quad=-i\left\langle\Psi\left(\theta_{1}, \theta_{2}, \phi_{1}, \cdots, \phi_{l}\right)\left|\partial_{\theta_{i}}\right| \Psi\left(\theta_{1}, \theta_{2}, \phi_{1}, \cdots, \phi_{l}\right)\right\rangle
\end{aligned}
$$

for $i=1,2$, and a first Chern number

$$
C\left(\phi_{1}, \cdots, \phi_{l}\right)=\frac{1}{2 \pi} \int_{0}^{2 \pi} d \theta_{1} d \theta_{2} F_{12}\left(\theta_{1}, \theta_{2}, \phi_{1}, \cdots, \phi_{l}\right) .
$$

Now, the $d$-dimensional Hall conductance is given by

$$
\begin{aligned}
\sigma_{d}= & \sum_{\phi_{1}, \cdots, \phi_{l}=\phi_{0}, 0}(-1)^{\sum_{i} \delta\left(\phi_{i}, 0\right)} C\left(\phi_{1}, \cdots, \phi_{l}\right) \\
= & C\left(\phi_{0}, \cdots, \phi_{0}, \phi_{0}\right)-C\left(\phi_{0}, \cdots, \phi_{0}, 0\right)+\cdots \\
& -C(0, \cdots, 0,0),
\end{aligned}
$$

where the delta function satisfies $\delta\left(\phi_{i}, 0\right)=1$ when $\phi_{i}=0$, and $\delta\left(\phi_{i}, 0\right)=0$ when $\phi_{i}=\phi_{0} \equiv 2 \pi$. When $d=$ 4 (i.e., $l=1$ ), Eq. (24) reduces to Eq. (12). The original Niu-Thouless-Wu formula is also a special case of Eq. (24) with $d=2$ (i.e., $l=0$ ).

\section{FRACTIONAL QUANTUM HALL EFFECTS}

One of the main motivations for introducing the topological invariant in Eq. (12) is its potential applications in fractional quantum Hall states. Before moving to higher dimensions, let us first present a review of the NiuThouless-Wu formula of $2 \mathrm{D}$ fractional $\mathrm{QH}$. As has been known in Ref. [77], fractional quantization of 2D Hall conductance is possible if the ground states are degenerate on a 2D torus.

In 2D, the standard boundary condition is given [77] by Eq. (5) except that the argument $\phi$ is absent. Suppose that a 2D fractional quantum Hall system has $p$-fold degenerate ground states $\left|\Psi_{1}\left(\theta_{1}, \theta_{2}\right)\right\rangle, \cdots,\left|\Psi_{p}\left(\theta_{1}, \theta_{2}\right)\right\rangle$ [86]. The Hall conductance is given by an average over these degenerate ground states as [77] (recall that we have taken the units $e=h=c=1$ )

$$
\begin{aligned}
\sigma_{2 D} & =\frac{1}{p} \frac{1}{2 \pi} \int_{0}^{2 \pi} d \theta_{1} d \theta_{2} \sum_{\alpha=1}^{p}\left[\left\langle\partial_{\theta_{1}} \Psi_{\alpha} \mid \partial_{\theta_{2}} \Psi_{\alpha}\right\rangle-\left\langle\partial_{\theta_{2}} \Psi_{\alpha} \mid \partial_{\theta_{1}} \Psi_{\alpha}\right\rangle\right] \\
& =\frac{1}{p} \frac{1}{2 \pi} \int_{0}^{2 \pi} d \theta_{1} d \theta_{2} \operatorname{Tr} F_{12}\left(\theta_{1}, \theta_{2}\right) \\
& =\bar{C}_{1}
\end{aligned}
$$

where the matrix elements of the non-Abelian Berry curvature $F_{i j}$ read $F_{i j}^{\alpha \beta}=\partial_{i} a_{j}^{\alpha \beta}-\partial_{j} a_{i}^{\alpha \beta}+i\left[a_{i}, a_{j}\right]^{\alpha \beta}$, in which $a_{i}^{\alpha \beta}=-i\left\langle\Psi_{\alpha}\left(\theta_{1}, \theta_{2}\right)\left|\partial_{\theta_{i}}\right| \Psi_{\beta}\left(\theta_{1}, \theta_{2}\right)\right\rangle$ is the non-Abelian Berry connection. The average Chern number $\bar{C}_{1} \equiv \frac{1}{p} C_{1} \equiv \frac{1}{p} \frac{1}{2 \pi} \int_{0}^{2 \pi} d \theta_{1} d \theta_{2} \operatorname{Tr} F_{12}\left(\theta_{1}, \theta_{2}\right)$, where $C_{1}$ is the standard definition of the first Chern number [87] of the $U(p)$ fiber bundle. Note that the $i\left[a_{i}, a_{j}\right]$ term in $F_{i j}$ vanishes after the tracing. It is a mathematical fact that the first Chern number $C_{1}$ is quantized as an integer; therefore, the Hall conductance is quantized as a rational number with denominator $p$.

Equation (25) can be rewritten as [77]

$$
\begin{aligned}
\sigma_{2 \mathrm{D}}= & \frac{1}{p} \frac{1}{2 \pi i} \int_{0}^{2 \pi p} d \theta_{1} \int_{0}^{2 \pi} d \theta_{2}\left[\left\langle\partial_{\theta_{1}} \Psi_{1} \mid \partial_{\theta_{2}} \Psi_{1}\right\rangle\right. \\
& \left.-\left\langle\partial_{\theta_{2}} \Psi_{1} \mid \partial_{\theta_{1}} \Psi_{1}\right\rangle\right]
\end{aligned}
$$

where we have picked up a ground state $\Psi_{1}$ from the degenerate ground state $\Psi_{1}, \cdots, \Psi_{p}$. The parameter space has been enlarged to $\left(0<\theta_{1}<2 \pi p, 0<\theta_{2}<2 \pi\right)$.

Now, let us move to higher dimensions. For a 4D fractional QH system, suppose that the ground states are $p$-fold degenerate on the 4D torus $T^{4}$ with boundary conditions described in Sec. II; in other words, the ground states form a $U(p)$ bundle over the $2 \mathrm{D}$ torus with coordinates $\left(\theta_{1}, \theta_{2}\right)$ (note that $\phi$ is fixed) [88]. We can define the Berry connection $a_{i}^{\alpha \beta}\left(\theta_{1}, \theta_{2}, \phi\right)=-i\left\langle\Psi_{\alpha}\left(\theta_{1}, \theta_{2}, \phi\right)\left|\partial_{\theta_{i}}\right| \Psi_{\beta}\left(\theta_{1}, \theta_{2}, \phi\right)\right\rangle \quad$ and the Berry curvature $F_{i j}^{\alpha \beta}=\partial_{i} a_{j}^{\alpha \beta}-\partial_{j} a_{i}^{\alpha \beta}+i\left[a_{i}, a_{j}\right]^{\alpha \beta}$. Equation (11) can be straightforwardly generalized as

$$
C(\phi)=\frac{1}{2 \pi} \int_{0}^{2 \pi} d \theta_{1} d \theta_{2} \operatorname{Tr} F_{12}\left(\theta_{1}, \theta_{2}, \phi\right) .
$$

Note that in Sec. II, we considered nondegenerate ground states; therefore, the symbol "Tr" in Eq. (27) is absent in Eq. (11). We can also define the average (first) Chern number for $4 \mathrm{D} \mathrm{QH}$ as

$$
\bar{C}(\phi)=C(\phi) / p .
$$

By analogy with Eq. (25), the 4D Hall conductance $\sigma_{4 \mathrm{D}}$ for fractional quantum Hall effects is obtained as

$$
\sigma_{4 \mathrm{D}}=\bar{C}\left(\phi_{0}\right)-\bar{C}(0) .
$$

Equation (29) is among the central results of this paper. In the presence of time-reversal symmetry, the second term vanishes. Equation (29) reduces to Eq. (12) when $p=1$, namely, the case without ground-state degeneracy.

To conclude this section, we mention that the generalization of Eq. (24) for $d=2 l+2$-dimensional fractional states reads $\sigma_{d}=\sum_{\phi_{1}, \cdots, \phi_{l}=\phi_{0}, 0}(-1)^{\sum_{i} \delta\left(\phi_{i}, 0\right)} \bar{C}\left(\phi_{1}, \cdots, \phi_{l}\right)$.

\section{MORE TOPOLOGICAL INVARIANTS FOR 4D FRACTIONAL QH}

Having studied the 4D fractional Hall conductance using the $\left(\theta_{1}, \theta_{2}, \phi\right)$ boundary conditions that we have chosen, let us investigate other choices of boundary conditions. The simplest choice is 


$$
\begin{aligned}
& \Psi\left(\mathbf{r}_{1}, \cdots, \mathbf{r}_{k}+L_{i} \hat{\mathbf{x}}_{i}, \cdots, \mathbf{r}_{N} ; \theta_{1}, \theta_{2}, \theta_{3}, \theta_{4}\right) \\
& \quad=\exp \left(i \theta_{i}\right) \Psi\left(\mathbf{r}_{1}, \cdots, \mathbf{r}_{k}, \cdots, \mathbf{r}_{N} ; \theta_{1}, \theta_{2}, \theta_{3}, \theta_{4}\right)
\end{aligned}
$$

for $i=1,23,4$. Suppose that the ground states are $p$-fold degenerate; then, these ground states form a $U(p)$ fiber bundle on the $4 \mathrm{D}$ torus parametrized by $\left(\theta_{1}, \theta_{2}, \theta_{3}, \theta_{4}\right)$, with $0 \leq \theta_{i}<2 \pi$. We can define a natural topological invariant

$$
C_{2}=\frac{1}{32 \pi^{2}} \int d^{4} \theta \epsilon^{i j k l} \operatorname{Tr} F_{i j} F_{k l}
$$

where the matrix elements of non-Abelian Berry curvature $F_{i j}$ are defined as $F_{i j}^{\alpha \beta}\left(\theta_{1}, \theta_{2}, \theta_{3}, \theta_{4}\right)=\partial_{i} a_{j}^{\alpha \beta}-\partial_{j} a_{i}^{\alpha \beta}+$ $i\left[a_{i}, a_{j}\right]^{\alpha \beta}$, where $i, j=1,2,3,4$. Equation (31) is a second Chern number defined for fractional $\mathrm{QH}$ states in $4 \mathrm{D}$. It should not be confused with the (lowercase) $c_{2}$ in Eq. (2), which is defined in terms of the free Bloch states of noninteracting systems.

For $2 n$-dimensional quantum Hall effects, we can straightforwardly generalize $C_{2}$ to $C_{n}$ as

$$
\begin{aligned}
C_{n} & =\frac{1}{n !} \int \operatorname{Tr}\left(\frac{F}{2 \pi}\right)^{n} \\
& =\frac{1}{2^{n} n !(2 \pi)^{n}} \int d^{2 n} \theta \epsilon^{\alpha_{1} \cdots \alpha_{2 n}} \operatorname{Tr} F_{\alpha_{1} \alpha_{2}} \cdots F_{\alpha_{2 n-1} \alpha_{2 n}},
\end{aligned}
$$

which are topological invariants for higher-dimensional fractional $\mathrm{QH}$ states.

In $2 \mathrm{D}$, the first Chern number $C_{1}$ of the $U(p)$ bundle is proportional to the Hall conductance $\sigma_{2 D}$. In fact, Eq. (25) tells us that $C_{1}=p \sigma_{2 D}$; thus, $C_{1}$ does not give us new topological invariants other than $\sigma_{2 D}$ and $p$. However, the 4D case is quite different. The key difference between $2 \mathrm{D}$ and $4 \mathrm{D}$ is as follows. For the $2 \mathrm{D} \mathrm{QH}$, both $\sigma_{2 D}$ and $C_{1}$ are defined under the same boundary condition parametrized by $\left(\theta_{1}, \theta_{2}\right)$. For $4 \mathrm{D}$ quantum Hall insulators, the topological invariants $C_{2}$ and $\sigma_{4 D}$ are defined using different boundary conditions [Eqs. $(5,6)$, and (7) for $\sigma_{4 \mathrm{D}}$, but Eq. (30) for $C_{2}$ ]; therefore, there is no direct relation between $C_{2}$ and $\sigma_{4 D}$. In principle, $C_{2}$ can take different values, given the same value of ground-state degeneracy $p$ and Hall coefficient $\sigma_{4 D}$. The topological invariant $C_{2}$ suggests that there are rich structures in 4D quantum Hall effects. Higher-dimensional QHs are similar: Higher Chern numbers $C_{n}(n=2,3, \cdots)$ are not directly related to the Hall coefficient $\sigma_{d}$ because they are defined under different boundary conditions.

\section{TOPOLOGICAL INSULATORS IN ONE DIMENSION}

In this section, we will briefly discuss 1D topological insulators to prepare for the investigation of 3D topological insulators in Sec. VIII. One-dimensional topological insulators can be characterized by a $\theta$ term [11]

$$
S_{\mathrm{eff}}=\frac{1}{2 \pi} \int d t d x \epsilon^{\mu \nu} \theta \partial_{\mu} A_{\nu}
$$

Let us study the $1 \mathrm{D}$ insulator on a torus $T^{1}$, which is just a circle. We take the boundary condition as

$$
\begin{aligned}
& \Psi\left(\mathbf{r}_{1}, \cdots, \mathbf{r}_{k}+L_{1} \hat{\mathbf{x}}_{1}, \cdots, \mathbf{r}_{N} ; \theta_{1}\right) \\
& \quad=\exp \left(i \theta_{1}\right) \Psi\left(\mathbf{r}_{1}, \cdots, \mathbf{r}_{k}, \cdots, \mathbf{r}_{N} ; \theta_{1}\right)
\end{aligned}
$$

namely, there is a gauge potential $A_{1}=\theta_{1} / L_{1}$.

Now, there exists a simple topological invariant [89,29]

$$
\Gamma=\int_{0}^{2 \pi} d \theta_{1} a_{1}\left(\theta_{1}\right)
$$

where the Berry connection is defined as $a_{1}\left(\theta_{1}\right)=$ $-i\left\langle\Psi\left(\theta_{1}\right)\left|\partial_{\theta_{1}}\right| \Psi\left(\theta_{1}\right)\right\rangle$. Equation (35) is an interacting generalization of the Zak phase [90]. It has been applied to 1D models [29,30], though its relation to the $\theta$ term was not discussed. Equation (35) is defined modulo $2 \pi$ because a local gauge transformation of the wave function can change it by $2 \pi$.

When the ground state $\left|\Psi\left(\theta_{1}\right)\right\rangle$ is not degenerate, the $\theta$ value is given by $\theta=\Gamma$. Since we are mainly concerned with higher-dimensional topological insulators, we will not study applications of this $1 \mathrm{D}$ formula in detail. It is useful to mention that the quantity $\partial \theta / \partial \lambda$, where $\lambda$ is a tuning parameter of the many-body Hamiltonian, is usually more useful than $\theta$ itself because $\partial \theta / \partial \lambda$ does not have any ambiguity under local gauge transformation of wave functions [89].

When the ground states are $p$-fold degenerate, the natural generalization of Eq. (35) is

$$
\Gamma=\int d \theta_{1} \operatorname{Tr} a_{1}\left(\theta_{1}\right)
$$

where the non-Abelian gauge potential is defined as $a_{1}^{\alpha \beta}=-i\left\langle\Psi_{\alpha}\left(\theta_{1}\right)\left|\partial_{\theta_{1}}\right| \Psi_{\beta}\left(\theta_{1}\right)\right\rangle$. The $\theta$ angle in Eq. (33) is given by

$$
\theta=\bar{\Gamma}
$$

where the average $\bar{\Gamma}$ is defined as $\bar{\Gamma}=\frac{1}{p} \Gamma$.

\section{TOPOLOGICAL INSULATORS IN THREE DIMENSIONS: INTEGER AND FRACTIONAL}

The approach we applied to $4 \mathrm{D} \mathrm{QH}$ states can be naturally generalized to $3 \mathrm{D}$. The $3 \mathrm{D}$ boundary conditions are chosen as follows. First,

$$
\begin{aligned}
& \Psi\left(\mathbf{r}_{1}, \cdots, \mathbf{r}_{k}+L_{1} \hat{\mathbf{x}}_{1}, \cdots, \mathbf{r}_{N} ; \theta_{1}, \phi\right) \\
& \quad=\exp \left(i \theta_{1}\right) \Psi\left(\mathbf{r}_{1}, \cdots, \mathbf{r}_{k}, \cdots, \mathbf{r}_{N} ; \theta_{1}, \phi\right)
\end{aligned}
$$


where $\mathbf{r}_{k}$ is the coordinate of the $k$ th particle (other variables such as spin are not shown for simplicity of notation), and $\hat{\mathbf{x}}_{1}$ is the unit vector along the $x_{1}$ direction. Second,

$$
\begin{aligned}
& \Psi\left(\mathbf{r}_{1}, \cdots, \mathbf{r}_{k}+L_{2} \hat{\mathbf{x}}_{2}, \cdots, \mathbf{r}_{N} ; \theta_{1}, \phi\right) \\
& \quad=\exp \left(-i \phi \frac{x_{3}}{L_{3}}\right) \Psi\left(\mathbf{r}_{1}, \cdots, \mathbf{r}_{k}, \cdots, \mathbf{r}_{N} ; \theta_{1}, \phi\right)
\end{aligned}
$$

and

$$
\begin{gathered}
\Psi\left(\mathbf{r}_{1}, \cdots, \mathbf{r}_{k}+L_{3} \hat{\mathbf{x}}_{3}, \cdots, \mathbf{r}_{N} ; \theta_{1}, \phi\right) \\
=\Psi\left(\mathbf{r}_{1}, \cdots, \mathbf{r}_{k}, \cdots, \mathbf{r}_{N} ; \theta_{1}, \phi\right),
\end{gathered}
$$

where $\phi$ satisfies the same quantization condition as discussed in Sec. II. Now, the $\theta$ angle in Eq. (3) is proposed (for the cases without ground-state degeneracy) as

$$
\theta=\Gamma\left(\phi_{0}\right)-\Gamma(0)
$$

where $\phi_{0} \equiv 2 \pi$, and

$$
\Gamma(\phi)=\int_{0}^{2 \pi} d \theta_{1} a_{1}\left(\theta_{1}, \phi\right),
$$

with $a_{1}\left(\theta_{1}, \phi\right)=-i\left\langle\Psi\left(\theta_{1}, \phi\right)\left|\partial_{\theta_{1}}\right| \Psi\left(\theta_{1}, \phi\right)\right\rangle$ being the Berry connection defined in terms of the ground-state wave function. One can derive Eq. (41) by calculating the Berry phase gained by the adiabatic evolution $A_{1} \rightarrow A_{1}+2 \pi / L_{1}$. Because of the topological terms $\frac{\theta}{4 \pi^{2}} \partial_{0} A_{1}\left(\partial_{2} A_{3}-\partial_{3} A_{2}\right)$ contained in the $\theta$ term, when a flux $\phi$ exists in $T_{23}$, as Eqs. (39) and (40) indicate, the adiabatic evolution of $A_{1} \rightarrow$ $A_{1}+2 \pi / L_{1}$ generates a topological phase $\theta \phi / 2 \pi$, which should be identified as the Berry phase accumulated by the adiabatic evolution of ground-state wave functions, namely, $\int d \theta_{1} a_{1}\left(\theta_{1}, \phi\right)$. It follows that Eq. (41) is the formula for $\theta$. Note that, potentially, there is another term $\theta^{\prime} \partial_{0} A_{1}$ that can contribute to the Berry phase in the evolution $A_{1} \rightarrow A_{1}+2 \pi / L_{1}$, which is the reason why the second term in Eq. (41) appears.

If the Hamiltonian and the ground state depend on a tuning parameter, which we denote as $\theta_{2}$, then $\theta$ is a function of $\theta_{2}$. The derivative of $\theta$ with respect to $\theta_{2}$ is given by the gauge-invariant formula

$$
\frac{\partial \theta}{\partial \theta_{2}}=\int_{0}^{2 \pi} d \theta_{1}\left[F_{21}\left(\theta_{1}, \theta_{2}, \phi_{0}\right)-F_{21}\left(\theta_{1}, \theta_{2}, 0\right)\right],
$$

where $F_{21}\left(\theta_{1}, \theta_{2}, \phi\right)=\partial_{\theta_{2}} a_{1}-\partial_{\theta_{1}} a_{2}$ and $a_{i}\left(\theta_{1}, \theta_{2}, \phi\right)=$ $-i\left\langle\Psi\left(\theta_{1}, \theta_{2}, \phi\right)\left|\frac{\partial}{\partial \theta_{i}}\right| \Psi\left(\theta_{1}, \theta_{2}, \phi\right)\right\rangle$. Similar to the $1 \mathrm{D}$ case discussed in Sec. VII, the quantity $\partial \theta / \partial \theta_{2}$ is usually more useful than $\theta$ itself because $\partial \theta / \partial \theta_{2}$ is invariant under any local gauge transformation of the wave function.

We will apply Eq. (41) to a noninteracting Dirac model in the Appendix, which gives the same result as obtained [11] from Eq. (4).
In the above calculations, we have assumed that the $\theta$ term is isotropic, which is always satisfied if there is time-reversal symmetry (though the Maxwell terms are generally still anisotropic). If the $\theta$ term is anisotropic [91,92], namely, if we have $\chi_{i j} E_{i} B_{j}=$ $\chi_{i j} E_{i} \epsilon_{j k l}\left(\partial_{k} A_{l}-\partial_{l} A_{k}\right)$, we should calculate each coefficient $\chi_{i j}$ separately, which is also given by Eq. (41) except that the twisted phase $\theta_{1}$ in Eq. (38) is added in the $x_{i}$ direction instead of the $x_{1}$ direction, and the flux $\phi$ [see Eqs. (39) and (40)] is added in the $\left(x_{k}, x_{l}\right)$ plane.

For 3D fractional states with $p$-fold ground-state degeneracy, we can generalize Eq. (41) as

$$
\theta=\bar{\Gamma}\left(\phi_{0}\right)-\bar{\Gamma}(0),
$$

where $\bar{\Gamma}(\phi) \equiv \frac{1}{p} \int_{0}^{2 \pi} d \theta_{1} \operatorname{Tr} a_{1}\left(\theta_{1}, \phi\right)$. The logic is similar to Sec. V. An important feature is notable here. We have the transformation rule $a_{1} \rightarrow U a_{1} U^{\dagger}+i U \partial U^{\dagger}$ under a local gauge transformation of the basis of ground-state wave functions, where $U=U\left(\theta_{1}, \phi\right)$ is a $p \times p$ unitary matrix. This may change $\Gamma(\phi)$ by multiples of $2 \pi / p$; therefore, the $\theta$ angle of fractional topological insulators is determined modulo $2 \pi / p$.

As a digression, let us briefly mention the generalization for a $d=2 l+1$-(spatial)-dimensional (isotropic) $\theta$ term if the system does not have ground-state degeneracy on a $d$-dimensional torus. The formula reads

$$
\begin{aligned}
\theta_{d}= & \sum_{\phi_{1}, \cdots, \phi_{l}=\phi_{0}, 0}(-1)^{\sum_{i} \delta\left(\phi_{i}, 0\right)} \Gamma\left(\phi_{1}, \cdots, \phi_{l}\right) \\
= & \Gamma\left(\phi_{0}, \cdots, \phi_{0}, \phi_{0}\right)-\Gamma\left(\phi_{0}, \cdots, \phi_{0}, 0\right)+\cdots \\
& -\Gamma(0, \cdots, 0,0),
\end{aligned}
$$

which is analogous to Eq. (24). The meanings of the arguments $\phi_{1}, \cdots, \phi_{l}$ are similar to that of Eq. (24), and we shall not repeat them here. If the state is fractional, we have $\theta_{d}=\sum_{\phi_{1}, \cdots, \phi_{l}=\phi_{0}, 0}(-1)^{\sum_{i} \delta\left(\phi_{i}, 0\right)} \bar{\Gamma}\left(\phi_{1}, \cdots, \phi_{l}\right)$, where $\bar{\Gamma}\left(\phi_{1}, \cdots, \phi_{l}\right) \equiv \Gamma\left(\phi_{1}, \cdots, \phi_{l}\right) / p$, the integer $p$ being the ground-state degeneracy.

\section{CONCLUSIONS}

In this paper, we have defined precise topological invariants in terms of the ground-state wave functions on a torus. This approach provides a conceptual framework in which many topological invariants and topologicalfield-theoretical coefficients, such as $\sigma_{4 D}$ (in 4D) and $\theta$ (in 3D), acquire precise definitions even in the presence of an arbitrary interaction and disorder.

Numerically, we do not expect that the wave function (on a torus) approach followed in the present paper will be as efficient as the topological Hamiltonian approach $[68,67]$ mentioned in Sec. I. However, the present approach has a wider range of validity because it is applicable in the 
presence of an arbitrary interaction and disorder; therefore, the present approach is highly desirable for certain purposes, especially when both the interaction and disorder are present, or when the interaction is so strong that exotic fractional states are generated. It is also useful to note that the topological invariants in the present paper can also be applied to bosonic topological insulators, for which other topological invariants are hard to define.

\section{ACKNOWLEDGMENTS}

Z. W. would like to thank Liang Kong and Yong-Shi Wu for helpful discussions. Z. W. is supported by NSFC under Grant No. 11304175. S.-C.Z. is supported by the Department of Energy, Office of Basic Energy Sciences, Division of Materials Sciences and Engineering, under Contract No. DE-AC02-76SF00515.

\section{APPENDIX: APPLICATION IN A THREE- DIMENSIONAL NONINTERACTING MODEL}

In the noninteracting limit, Eq. (41) should give the same $\theta$ as the noninteracting formula [11]. In this Appendix, we will check this result in a simple noninteracting model. This Appendix follows similar calculations as Sec. III.

Let us study a simple 3D noninteracting Dirac model given as

$h(\mathbf{k})=v \sin k_{1} \tau^{1}+\left[v \sin k_{2} \sigma^{1}+v \sin k_{3} \sigma^{2}+M(\mathbf{k}) \sigma^{3}\right] \tau^{3}$,

where $M(\mathbf{k})=m+3-\sum_{i=1}^{3} \cos k_{i}$. In the limit that $|m| \ll 1$, the low-energy physics is dominated by the $k \approx 0$ region, and we can linearly expand $h(\mathbf{k})$ as

$$
h(\mathbf{k}) \approx v k_{1} \tau^{1}+\left(v k_{2} \sigma^{1}+v k_{3} \sigma^{2}+m \sigma^{3}\right) \tau^{3} .
$$

The boundary conditions are given in Eqs. $(38,39)$, and (40), which means that there is a flux $\phi$ inside the $2 \mathrm{D}$ torus $T_{23}$. First, let us take $\phi=\phi_{0} \equiv 2 \pi$. By a calculation similar to Sec. III, we can first solve $h^{\prime}\left(k_{2}, k_{3}\right)=v k_{2} \sigma^{1}+v k_{3} \sigma^{2}+$ $m \sigma^{3}$ after replacing $k_{i} \rightarrow-i\left(\partial_{i}-A_{i}\right)$, whose eigenvalues read

$$
E_{0}=m ; \quad E_{n \pm}= \pm \sqrt{m^{2}+2 n B v^{2}}(n=1,2, \cdots),
$$

and the corresponding wave functions are $\left(\psi_{0}, 0\right)^{T}$ and $\left(\psi_{n}, \pm \psi_{n-1}\right)^{T}$. Now, we put these eigenvalues back into the set of parentheses of Eq. (A2); then, we have the 1D Hamiltonians

$$
\begin{aligned}
h_{0} & =v k_{1} \tau^{1}+m \tau^{3} \\
h_{n \pm} & =v k_{1} \tau^{1}+E_{n \pm} \tau^{3}(n=1,2, \cdots) .
\end{aligned}
$$

Now, the $\Gamma\left(\phi_{0}\right)$ in Eq. (41) can be found as $\frac{\pi}{2}\left[\operatorname{sgn}\left(E_{0}\right)+\sum_{n} \sum_{\alpha= \pm} \operatorname{sgn}\left(E_{n \alpha}\right)\right]=\frac{\pi}{2} \operatorname{sgn}(m)$, which is similar to Sec. III. Again, because of the high-energy regularization, we can only assert that $\Gamma\left(\phi_{0}\right)=$ $\frac{\pi}{2} \operatorname{sgn}(m)+$ constant. Consideration similar to in Sec. III leads to $\Gamma\left(\phi_{0}\right)=\frac{\pi}{2}(\operatorname{sgn}(m)-1)$. Similarly, we have $\Gamma(0)=0$; therefore, from Eq. (41) it follows that

$$
\theta=\Gamma\left(\phi_{0}\right)-\Gamma(0)=\frac{\pi}{2}(\operatorname{sgn}(m)-1)
$$

which means that $\theta=-\pi$ when $m<0$. This is consistent with the result obtained from the noninteracting ChernSimons term [11].

[1] X.-L. Qi and S.-C. Zhang, The Quantum Spin Hall Effect and Topological Insulators, Phys. Today 63, 33 (2010).

[2] M.Z. Hasan and C. L. Kane, Colloquium: Topological Insulators, Rev. Mod. Phys. 82, 3045 (2010).

[3] X.-L. Qi and S.-C. Zhang, Topological Insulators and Superconductors, Rev. Mod. Phys. 83, 1057 (2011).

[4] C. L. Kane and E. J. Mele, Quantum Spin Hall Effect in Graphene, Phys. Rev. Lett. 95, 226801 (2005).

[5] B. A. Bernevig and S. C. Zhang, Quantum Spin Hall Effect, Phys. Rev. Lett. 96, 106802 (2006).

[6] C. L. Kane and E. J. Mele, $Z_{2}$ Topological Order and the Quantum Spin Hall Effect, Phys. Rev. Lett. 95, 146802 (2005).

[7] B. A. Bernevig, T. L. Hughes, and S. C. Zhangt, Quantum Spin Hall Effect Topological Phase Transition in HgTe Quantum Wells, Science 314, 1757 (2006).

[8] M. König, S. Wiedmann, C. Brüne, A. Roth, H. Buhmann, L. Molenkamp, X.-L. Qi, and S.-C. Zhang, Quantum Spin Hall Insulator State in HgTe Quantum Wells, Science 318, 766 (2007).

[9] L. Fu and C. L. Kane, Time Reversal Polarization and $a Z_{2}$ Adiabatic Spin Pump, Phys. Rev. B 74, 195312 (2006).

[10] J. E. Moore and L. Balents, Topological Invariants of TimeReversal-Invariant Band Structures, Phys. Rev. B 75, 121306 (2007).

[11] X.-L. Qi, T. Hughes, and S.-C. Zhang, Topological Field Theory of Time-Reversal Invariant Insulators, Phys. Rev. B 78, 195424 (2008).

[12] L. Fu, C. L. Kane, and E. J. Mele,, Topological Insulators in Three Dimensions, Phys. Rev. Lett. 98, 106803 (2007).

[13] R. Roy, Topological Phases and the Quantum Spin Hall Effect in Three Dimensions, Phys. Rev. B 79, 195322 (2009).

[14] A. P. Schnyder, S. Ryu, A. Furusaki, and A. W. W. Ludwig, Classification of Topological Insulators and Superconductors in Three Spatial Dimensions, Phys. Rev. B 78, 195125 (2008).

[15] A. Kitaev, Periodic Table for Topological Insulators and Superconductors, Proceedings of the L. D. Landau Memorial Conference "Advances in Theoretical Physics", arXiv:0901.2686.

[16] S. Raghu, X.-L. Qi, C. Honerkamp, and S.-C. Zhang, Topological Mott Insulators Phys. Rev. Lett. 100, 156401 (2008). 
[17] A. Shitade, H. Katsura, J. Kune, X.-L. Qi, S.-C. Zhang, and N. Nagaosa, Quantum Spin Hall Effect in a Transition Metal Oxide $\mathrm{na}_{2} \mathrm{iro}_{3}$, Phys. Rev. Lett. 102, 256403 (2009).

[18] Y. Zhang, Y. Ran, and A. Vishwanath, Topological Insulators in Three Dimensions from Spontaneous Symmetry Breaking, Phys. Rev. B 79, 245331 (2009).

[19] B. Seradjeh, J. E. Moore, and M. Franz, Exciton Condensation and Charge Fractionalization in a Topological Insulator Film, Phys. Rev. Lett. 103, 066402 (2009).

[20] D. A. Pesin and L. Balents, Mott Physics and Band Topology in Materials with Strong Spin-Orbit Interaction, Nat. Phys. 6, 376 (2010).

[21] L. Fidkowski and A. Kitaev, Phys. Rev. B 81, 134509 (2010).

[22] M. Hohenadler, T. C. Lang, and F. F. Assaad, Correlation Effects in Quantum Spin-Hall Insulators: A Quantum Monte Carlo Study, Phys. Rev. Lett. 106, 100403 (2011).

[23] Z. Wang, X.-L. Qi, and S.-C. Zhang, Topological Order Parameters for Interacting Topological Insulators, Phys. Rev. Lett. 105, 256803 (2010).

[24] R. Li, J. Wang, X.-L. Qi, and S.-C. Zhang, Dynamical Axion Field in Topological Magnetic Insulators, Nat. Phys. 6, 284 (2010).

[25] M. Dzero, K. Sun, V. Galitski, and P. Coleman, Topological Kondo Insulators, Phys. Rev. Lett. 104, 106408 (2010).

[26] S. Rachel and K. Le Hur, Topological Insulators and Mott Physics from the Hubbard Interaction, Phys. Rev. B 82, 075106 (2010).

[27] N. Regnault and B. Andrei Bernevig, Fractional Chern Insulator, Phys. Rev. X 1, 021014 (2011).

[28] X. Zhang, H. Zhang, J. Wang, C. Felser, and S.-C. Zhang, Actinide Topological Insulator Materials with Strong Interaction, Science 335, 1464 (2012).

[29] H. Guo and S.-Q. Shen, Topological Phase in a OneDimensional Interacting Fermion System, Phys. Rev. B 84, 195107 (2011).

[30] H. Guo, S.-Q. Shen, and S. Feng, Fractional Topological Phase in One-Dimensional Flat Bands with Nontrivial Topology, Phys. Rev. B 86, 085124 (2012).

[31] D. N. Sheng, Z.-C. Gu, K. Sun, and L. Sheng, Fractional Quantum Hall Effect in the Absence of Landau Levels, Nat. Commun. 2, 389 (2011).

[32] D. Zheng, G.-M. Zhang, and C. Wu, Particle-Hole Symmetry and Interaction Effects in the Kane-MeleHubbard Model, Phys. Rev. B 84, 205121 (2011).

[33] S.-L. Yu, X.C. Xie, and J.-X. Li, Mott Physics and Topological Phase Transition in Correlated Dirac Fermions Phys. Rev. Lett. 107, 010401 (2011).

[34] Michael Levin and Ady Stern, Fractional Topological Insulators, Phys. Rev. Lett. 103, 196803 (2009).

[35] J. Maciejko, X.-L. Qi, A. Karch, and S.-C. Zhang, Fractional Topological Insulators in Three Dimensions, Phys. Rev. Lett. 105, 246809 (2010).

[36] B. Swingle, M. Barkeshli, J. McGreevy, and T. Senthil, Correlated Topological Insulators and the Fractional Magnetoelectric Effect, Phys. Rev. B 83, 195139 (2011).

[37] C. N. Varney, K. Sun, M. Rigol, and V. Galitski, Topological Phase Transitions for Interacting Finite Systems, Phys. Rev. B 84, 241105 (2011).
[38] C. Griset and C. Xu, Phase Diagram of the Kane-MeleHubbard Model, Phys. Rev. B 85, 045123 (2012).

[39] T. Neupert, L. Santos, S. Ryu, C. Chamon, and C. Mudry, Topological Hubbard Model and Its High-Temperature Quantum Hall Effect, Phys. Rev. Lett. 108, 046806 (2012).

[40] A. Go, W. Witczak-Krempa, G. Sang Jeon, K. Park, and Y. Baek Kim, Correlation Effects on 3D Topological Phases: From Bulk to Boundary, Phys. Rev. Lett. 109, 066401 (2012).

[41] X. Chen, Z.-C. Gu, Z.-X. Liu, and X.-G. Wen, Symmetry Protected Topological Orders and the Group Cohomology of Their Symmetry Group, Phys. Rev. B 87, 155114 (2013).

[42] X. Chen, Z.-C. Gu, Z.-X. Liu, and X.-G. Wen, Symmetry Protected Topological Orders in Interacting Bosonic Systems, arXiv:1301.0861.

[43] Z. Wang, X.-L. Qi, and S.-C. Zhang, Topological Field Theory and Thermal Responses of Interacting Topological Superconductors, Phys. Rev. B 84, 014527 (2011).

[44] J. C. Budich, R. Thomale, G. Li, M. Laubach, and S.-C. Zhang, Fluctuation-Induced Topological Quantum Phase Transitions in Quantum Spin-Hall and Anomalous-Hall Insulators, Phys. Rev. B 86, 201407 (2012).

[45] L. Wang, X. Dai, and X. C. Xie, Interaction-Induced Topological Phase Transition in the Bernevig-HughesZhang Model, Europhys. Lett. 98, 57001 (2012).

[46] Y.-F. Wang, Z.-C. Gu, C.-D. Gong, and D. N. Sheng, Fractional Quantum Hall Effect of Hard-Core Bosons in Topological Flat Bands, Phys. Rev. Lett. 107, 146803 (2011).

[47] M. Hohenadler, Z. Y. Meng, T. C. Lang, S. Wessel, A. Muramatsu, and F. F. Assaad, Quantum Phase Transitions in the Kane-Mele-Hubbard Model, Phys. Rev. B 85, 115132 (2012).

[48] M. Kargarian, J. Wen, and G. A. Fiete, Competing Exotic Topological Insulator Phases in Transition-Metal Oxides on the Pyrochlore Lattice with Distortion, Phys. Rev. B 83, 165112 (2011).

[49] F. F. Assaad, M. Bercx, and M. Hohenadler, Topological Invariant and Quantum Spin Models from Magnetic $\pi$ Fluxes in Correlated Topological Insulators, Phys. Rev. X 3, 011015 (2013).

[50] J. C. Budich, B. Trauzettel, and G. Sangiovanni, Fluctuation-Driven Topological Hund Insulators, Phys. Rev. B 87, 235104 (2013).

[51] H.-H. Hung, L. Wang, Z.-C. Gu, and G. A. Fiete, Topological Phase Transition in a Generalized Kane-Mele-Hubbard Model: A combined Quantum Monte Carlo and Green's Function Study, Phys. Rev. B 87, 121113 (2013).

[52] H.-H. Hung, V. Chua, L. Wang, and G. A. Fiete, Finite-Size and Interaction Effects on Topological Phase Transitions via Numerically Exact Quantum Monte Carlo Calculations, arXiv:1307.2659.

[53] J. Werner and F. F. Assaad, Interaction-Driven Transition between Topological States in a Kondo Insulator, Phys. Rev. B 88, 035113 (2013).

[54] F. Lu, J. Zhao, H. Weng, Z. Fang, and X. Dai, Correlated Topological Insulators with Mixed Valence, Phys. Rev. Lett. 110, 096401 (2013).

[55] T. C. Lang, A. M. Essin, V. Gurarie, and S. Wessel, $Z_{2}$ Topological Invariants in Two Dimensions from Quantum Monte Carlo, Phys. Rev. B 87, 205101 (2013). 
[56] M. Hohenadler and F. F. Assaad, Correlation Effects in Two-Dimensional Topological Insulators, J. Phys. Condens. Matter 25, 143201 (2013).

[57] X. Zhang, N. P. Butch, P. Syers, S. Ziemak, R. L. Greene, and J. Paglione, Hybridization, Inter-ion Correlation, and Surface States in the Kondo Insulator $\mathrm{SmB}_{6}$, Phys. Rev. X 3, 011011 (2013).

[58] L. Wang, H. Jiang, X. Dai, and X. C. Xie, Pole Expansion of Self-Energy and Interaction Effect for Topological Insulators, Phys. Rev. B 85, 235135 (2012).

[59] M. A. N. Araújo, E. V. Castro, and P. D. Sacramento, Change of an Insulator's Topological Properties by a Hubbard Interaction, Phys. Rev. B 87, 085109 (2013).

[60] S. C. Zhang and J. P. Hu, A Four-Dimensional Generalization of the Quantum Hall Effect, Science 294, 823 (2001).

[61] B. A. Bernevig, C. H. Chern, J. P. Hu, N. Toumbas, and S. C. Zhang, Ann. Phys. (N.Y.) 300, 185 (2002).

[62] B. I. Halperin, Quantized Hall Conductance, CurrentCarrying Edge States, and the Existence of Extended States in a Two-Dimensional Disordered Potential, Phys. Rev. B 25, 2185 (1982).

[63] X. G. Wen, Chiral Luttinger Liquid and the Edge Excitations in the Fractional Quantum Hall States, Phys. Rev. B 41, 12838 (1990).

[64] The lowercase letter, $c_{2}$ refers to the noninteracting limit, which should not be confused with the uppercase $C_{2}$ defined in Sec. VI.

[65] A. M. Essin, J. E. Moore, and D. Vanderbilt, Magnetoelectric Polarizability and Axion Electrodynamics in Crystalline Insulators, Phys. Rev. Lett. 102, 146805 (2009).

[66] Z. Wang, X.-L. Qi, and S.-C. Zhang, Equivalent Topological Invariants of Topological Insulators, New J. Phys. 12, 065007 (2010).

[67] Z. Wang and B. Yan, Topological Hamiltonian as an Exact Tool for Topological Invariants, J. Phys. Condens. Matter 25, 155601 (2013).

[68] Z. Wang and S.-C. Zhang, Simplified Topological Invariants for Interacting Insulators, Phys. Rev. X 2, 031008 (2012).

[69] Z. Wang and S.-C. Zhang, Strongly Correlated Topological Superconductors and Topological Phase Transitions via Green's Function, Phys. Rev. B 86, 165116 (2012).

[70] Z. Wang, X.-L. Qi, and S.-C. Zhang, Topological Invariants for Interacting Topological Insulators with Inversion Symmetry, Phys. Rev. B 85, 165126 (2012).

[71] S. Nakosai, J. C. Budich, Y. Tanaka, B. Trauzettel, and N. Nagaosa, Majorana Bound States and Nonlocal Spin Correlations in a Quantum Wire on an Unconventional Superconductor, Phys. Rev. Lett. 110, 117002 (2013).

[72] S. R. Manmana, A. M. Essin, R. M. Noack, and V. Gurarie, Topological Invariants and Interacting One-Dimensional Fermionic Systems, Phys. Rev. B 86, 205119 (2012).

[73] X. Deng, K. Haule, and G. Kotliar, Plutonium Hexaboride Is a Correlated Topological Insulator, Phys. Rev. Lett. 111, 176404 (2013).

[74] T. Yoshida, R. Peters, S. Fujimoto, and N. Kawakami, Topological Phase in a Two-Dimensional Metallic HeavyFermion System, Phys. Rev. B 87, 165109 (2013).
[75] J. C. Budich and B. Trauzettel, From the Adiabatic Theorem of Quantum Mechanics to Topological States of Matter, Phys. Status Solidi RRL 7, 109 (2013).

[76] Enlarging the unit cell can partially overcome this difficulty, which enables an extension of this approach to disordered systems.

[77] Q. Niu, D. J. Thouless, and Y.-S. Wu, Quantized Hall Conductance as a Topological Invariant, Phys. Rev. B 31, 3372 (1985).

[78] Note that there are two ways to define the twisted boundary condition. The first is to put the twisted phase factor $\left(\theta_{1}, \theta_{2}, \phi\right)$ in the wave function, as we did in Eqs. $(5,6)$, and (7). The second way is to add phase parameters in the Hamiltonian instead of the wave function. These two ways are equivalent and can be translated into each other. In fact, a gauge transformation of the wave function changes the twisted boundary condition to the periodic boundary condition, at the price of adding the twisted phase factor to the Hamiltonian.

[79] This is analogous to the Kaluza-Klein compactification.

[80] Wecan also write $\sigma_{4 D}=\left[C\left(n \phi_{0}\right)-C(0)\right] / n$ for any integer $n$. For simplicity, this will not be pursued in the present paper.

[81] D. J. Thouless, M. Kohmoto, M. P. Nightingale, and M. den Nijs, Quantized Hall Conductance in a Two-Dimensional Periodic Potential, Phys. Rev. Lett. 49, 405 (1982).

[82] R. B. Laughlin, Quantized Hall Conductivity in Two DimensionsPhys. Rev. B 23, 5632 (1981).

[83] A. H. Castro Neto, F. Guinea, N. M. R. Peres, K. S. Novoselov, and A. K. Geim, The Electronic Properties of Graphene, Rev. Mod. Phys. 81, 109 (2009).

[84] Y. Li, S.-C. Zhang, and C. Wu, Topological Insulators with SU(2) Landau Levels, Phys. Rev. Lett. 111, 186803 (2013).

[85] S. Ryu, A. Schnyder, A. Furusaki, and A. Ludwig, Topological Insulators and Superconductors: Ten-fold Way and Dimensional Hierarchy, New J. Phys. 12, 065010 (2010).

[86] It is worth noting that such a basis $\left\{\left|\Psi_{1}\right\rangle, \cdots,\left|\Psi_{p}\right\rangle\right\}$ can be found only locally, namely, that they can be defined only in a topologically trivial patch on the $2 \mathrm{D}$ torus parametrized by $\left(\theta_{1}, \theta_{2}\right)$. The quantities we actually need, such as $\operatorname{Tr} F_{12}\left(\theta_{1}, \theta_{2}\right)$, are independent of the basis choices.

[87] M. Nakahara, Geometry, Topology, and Physics (Bristol, Philadelphia, 1990).

[88] For simplicity, we assume that the degenerate ground states cannot be divided into smaller subspaces, with each subspace forming a fiber bundle over the torus parametrized by $\left(\theta_{1}, \theta_{2}\right)$. Otherwise, we can just pick up one subspace that cannot be reduced further into smaller subspaces, and everything discussed in this section will be unchanged.

[89] G. Ortiz and R. M. Martin, Macroscopic Polarization as a Geometric Quantum Phase: Many-Body Formulation, Phys. Rev. B 49, 14202 (1994).

[90] J. Zak, Quantization of Particle Transport, Phys. Rev. Lett. 62, 2747 (1989).

[91] A. M. Essin, A. M. Turner, J. E. Moore, and D. Vanderbilt, Orbital Magnetoelectric Coupling in Band Insulators, Phys. Rev. B 81, 205104 (2010).

[92] A. Malashevich, I. Souza, S. Coh, and D. Vanderbilt, Theory of Orbital Magnetoelectric Response, New J. Phys. 12, 053032 (2010). 